\title{
Analog Recording on Phase-change Optical Disks
}

\author{
Naoyasu Miyagawa* and Masud Mansuripur \\ Optical Sciences Center, The University of Arizona, Tucson, Arizona 85721, USA \\ *Permanent address: Matsushita Electrical Industrial Co., Ltd., Moriguchi, Osaka, 570-8501, Japan \\ e-mail: miyagawa@ctmo.mei.co.jp
}

( Received: Nov. 1, 2000, Accepted: Dec. 22, 2000 )

\begin{abstract}
Conventional optical recording methods strive to write a pattern of "perfect marks" on the media, then process the resulting read waveform electronically (using filters, equalizers, slicers) to extract the lengths/positions of the recorded marks. We propose to invert this process by concentrating at first on the read process, deciding which waveforms the read channel can and should supply given its particular constraints (e.g., spectral content, bandwidth, noise characteristics, restrictions of the peak amplitude, etc.). Once the desired read waveforms have been identified, we suggest strategies for recording "patterns" (e.g., amorphous/crystalline regions on phasechange media, magnetic domains on MO media) that can produce the desired waveforms during readout. By employing the heretofore forbidden mark/space patterns at the medium, the proposed analog schemes should be capable of delivering higher recording densities. Also, by allowing the system designer to match the read waveforms to channel characteristics, the proposed schemes should use the available SNR more efficiently.
\end{abstract}

Key words: phase-change optical disk, analog recording, write strategy, partial response, genetic algorithm.

\section{Introduction}

Looking back at optical data storage technology, perhaps the most significant breakthrough in this field has been a shift from analog to digital recording. ${ }^{1)}$ Due to the introduction of digital schemes, we have obtained many benefits such as error correction, data compression, etc. It must be recognized, however, that we have lost delicate features in the recorded patterns of information on the media. To eliminate the gap between digital and analog schemes and to increase recording capacity, various multilevel recording methods have been investigated. $\left.{ }^{2-5}\right)$

The tradition in digital storage is to try to record "perfect marks" on the storage media (disk, tape, etc.). An ideal mark is one that is fully saturated in one or the other state (e.g., amorphous/crystalline), fills the width of the track uniformly, has smooth boundaries, and has the correct length and center position. Writing ideal marks entails substantial efforts on the drive/ media designer. In this paper we propose alternative approaches to optical recording that relieve these burdens by combin-ing analog recording with digital processing schemes that preserve the main advantages of digital encoding while offering the potential of increased storage density.

\section{Segmented recording and matched-filter detection}

\subsection{Basic concept}

The proposed method is based on recording one byte at a time. A stream of binary data is divided into 8-bit segments, and each segment is written by a specific write pattern. The number of distinct write patterns is 256 , and each pattern realizes one of 256 different read waveforms. During readout, the waveforms obtained from individual segments are compared with waveforms in a pre-stored table, each element of which corresponds to a single byte of information. Using pattern-matching techniques, the recorded information can be retrieved.

As shown in Fig. 1, the length of each segment along the track is divided into two parts: One is the data block where the byte symbol is written; the other is the guard band that is left undisturbed. The guard band is needed to avoid inter-symbol interference (ISI) during both reading and writing. By using pattern-matching techniques to detect the readout waveform, the need for written marks to be oval shape and regular (i.e., ideal marks) is obviated.

The length of the guard band should be carefully optimized. Because we target the same linear density (without overhead) as DVD-ROM using a commercial DVD-RAM/2.6GB disk, the total segment length is set to $2.2 \mu \mathrm{m}$. Improvement of recording density is a factor of 1.55. The the guard band should be longer than the optical cutoff period of $\lambda /(2 \mathrm{NA}) \sim 0.55 \mu \mathrm{m}$. According to our thermal simulations, a $0.6 \mu \mathrm{m}$ guard band is also adequate for suppressing thermal interference.
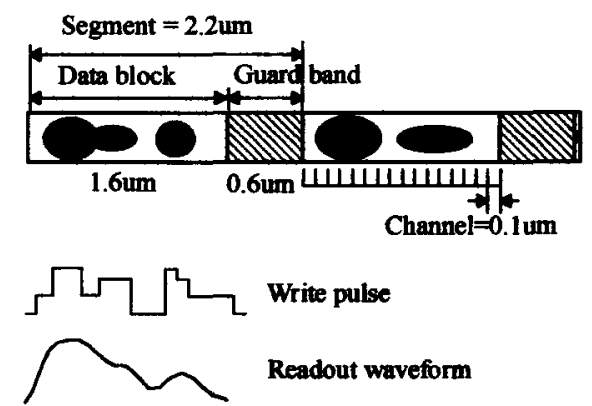

Figure 1. Diagram showing the data format, write pulse, and readout waveform of segmented analog recording.

\subsection{Constructing a waveform table}

To obtain 256 distinct read waveforms, various patterns of write pulses were attempted. These experiments were performed on a dynamic testbed ${ }^{6)}$ with $\lambda=660 \mathrm{~nm}$ and $\mathrm{NA}=0.6$ at $6 \mathrm{~m} / \mathrm{s}$. Each segment was divided into 22 channels: 16 channels for the data block and 6 channels for the guard band. The duration of each channel corresponds to $0.1 \mu \mathrm{m}$ or $17 \mathrm{~ns}$. If we do not use 4 of the channels during writing to avoid the extension of the recorded marks into the guard band, arbitrary laser power levels can be assigned to each of the 12 remaining 


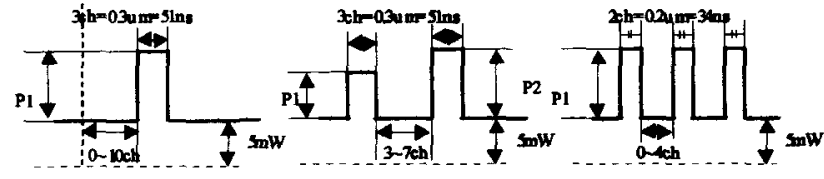

Figure 2. Write patterns containing one, two and three high pulses used in the recording experiments.
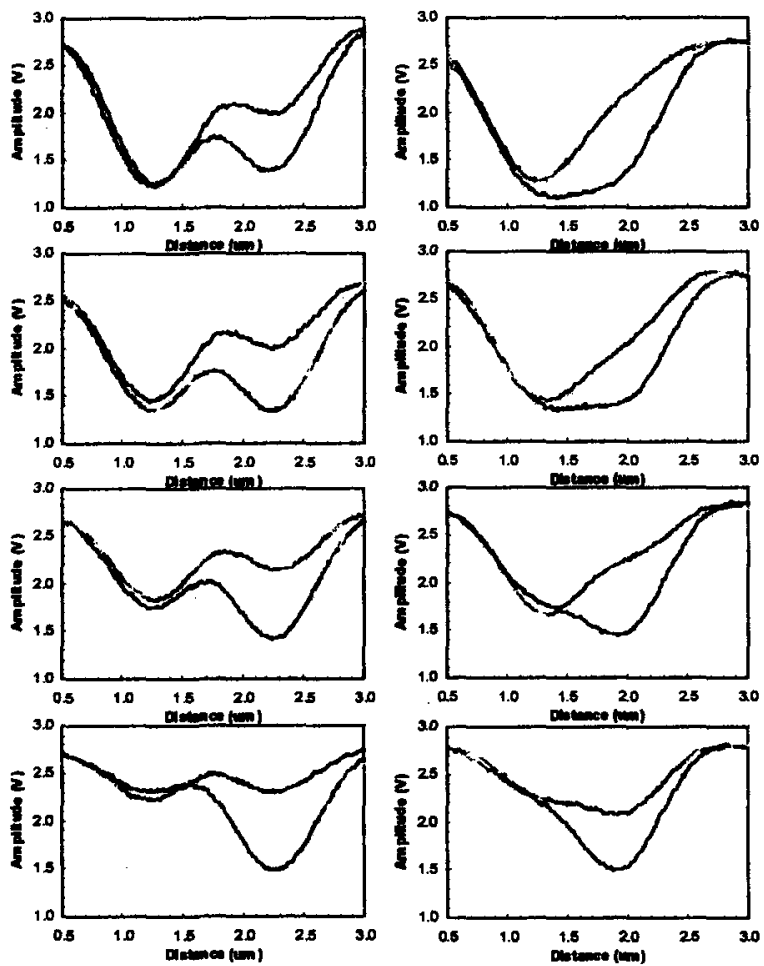

Figure 3. A portion of waveform table. Amorphous marks were recorded by write patterns with two high pulses. Intervals of pulses were $7 \mathrm{ch}$ (left) and 4ch (right). From top to bottom, $P_{1}$ was fixed at $14,12,10$ and $9 \mathrm{~mW}$ and $P_{2}$ was varied from 14 to $9 \mathrm{~mW}$ in each figure.

channels. Even with 3 laser power levels, we have $3^{12}=531441$ different write patterns to select from in search of our desired 256 distinct read patterns.

Next, we pursued a strategy of using simple write patterns that yield the targeted number of read waveforms. One could choose the write patterns randomly to obtain a large number of arbitrary read waveforms. However, we tried to produce a variety of waveforms in a systematic way by selecting one, two, or three pulses per segment, each pulse having one of 4 power levels and varying its position, as shown in Fig. 2. Our desire was to avoid complex algorithms for determining the write pattern given the facts that the choice of write pattern for a given read waveform was not unique, and that a certain amount of write power margin was necessary to distinguish the resulting waveforms from each other.

Numerous write patterns were subsequently used to record amorphous marks of differing shapes. Each read signal was then analyzed in terms of distinguishability from other waveforms. Fig. 3 shows some of the resulting waveforms that constitute our pattern table. A variety of patterns are seen to emerge. In our experiments the total number of waveforms obtained was 220 , which was somewhat below the desired 256. We believe, however,

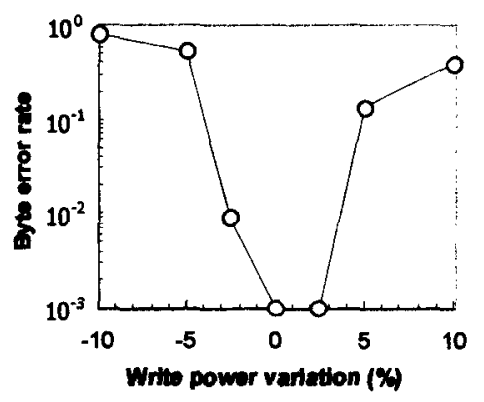

Figure 4. Dependence of the estimated byte error rate on write power fluctuations using simple pattern matching.

that this target would be attainable if more complex write patterns were introduced.

\subsection{Detection of readout waveforms}

Detecting readout waveforms is the second task of the proposed method. We prepared write pulses chosen randomly from our pattern table. The detection procedure was carried out using a digital oscilloscope and software tools. The cross-correlation coefficients between each readout segment and the patterns in the look-up table were calculated. Since cross-correlation could not distinguish waveforms with similar shapes but different magnitudes, the DC levels of the readout waveforms were also compared with those of the patterns in the table. When both parameters fell within acceptable ranges, the read pattern was considered to be successfully detected.

Fig. 4 shows the dependence of error-rate on write power variations around its nominal value. Although the error rate was below $10^{-3}$ at the optimum power level, it increased dramatically when the write power decreased by more than $5 \%$. Although the achieved improvement of the recording density was a factor of 1.55 or less, we should conclude that precise laser power control is indispensable for such a simple pattern matching detection scheme.

\section{Analog Partial Response Method}

\subsection{Basic concept}

The preceding section provided an outlook on the capabilities of the analog recording. However, since the method requires a guard-band, its storage efficiency is rather poor. Moreover, signal detection by matched filtering on a large scale may not be affordable for practical use. Thus we decided to improve it by employing the PRML signaling schemes.

We begin by identifying ideal read waveforms that match the channel characteristics as closely as possible. Because these waveforms are expected to emerge directly from the photo-detector, they require no further (electronic) equalization, thus eliminating the additional noise introduced by equalization in conventional systems. The spectrum of our readout waveforms can be made to follow the channel's SNR spectrum. This allows a closer approach to the information-theoretic capacity of the media than is possible in conventional systems, where the read waveform is a by-product of a writing strategy that is largely ignorant of the read channel characteristics. 


\subsection{Selecting the Read Waveforms}

We begin by choosing a pair of waveforms for the individual channel bits: One and Zero. Such a pair may resemble those in Figs. $7(a, b)$, and should match the channel characteristics as closely as possible. Typically these functions are zero at all multiples of the clock period $\Delta$, except at a finite number of points, where they have well-defined non-zero values. (In Fig.5, the non-zero values of the chosen functions are \pm 0.75 and \pm 1.5 volts, occurring at $t=2 \Delta, 3 \Delta$ and $4 \Delta$. Note that the Zero waveform is simply an inverted version of the One waveform.) Since our One/Zero waveforms are similar to the equalized output of a PRML channel, Viterbi's algorithm can be used at the end to extract the stored bitsequences from the readout waveforms.

The clock period $\Delta$ is chosen based on the wavelength $\lambda$ of the laser, the numerical aperture NA of the objective, and the linear velocity $V$ of the disk. In the example of Fig.5, the width of the individual One/Zero waveforms, $\sim 4 \Delta$, must be roughly equal to $\lambda$ (NA. $\eta$.

The One and Zero waveforms of Fig.5 have identical spectra (because they differ only by a sign factor). The normalized amplitude of the Fourier transform of these functions is shown on a linear scale in Fig.5(c), and on a logarithmic scale (decibels) in Fig.5(d). The cutoff is seen to occur at $f \Delta \approx 0.5$, corresponding to $f_{\mathrm{c}}=2 \mathrm{NAV} / \lambda$, which is the well-known cutoff frequency of optical storage systems. Above the cutoff, the spectral strength of the waveform is less than $-36 \mathrm{~dB}$, which is typically below the noise level and, therefore, acceptable.

During readout, the spectrum of a random bit-sequence will be the same as the Fourier transform of the One/Zero waveform, shown in Fig.5(d). Therefore, in the initial
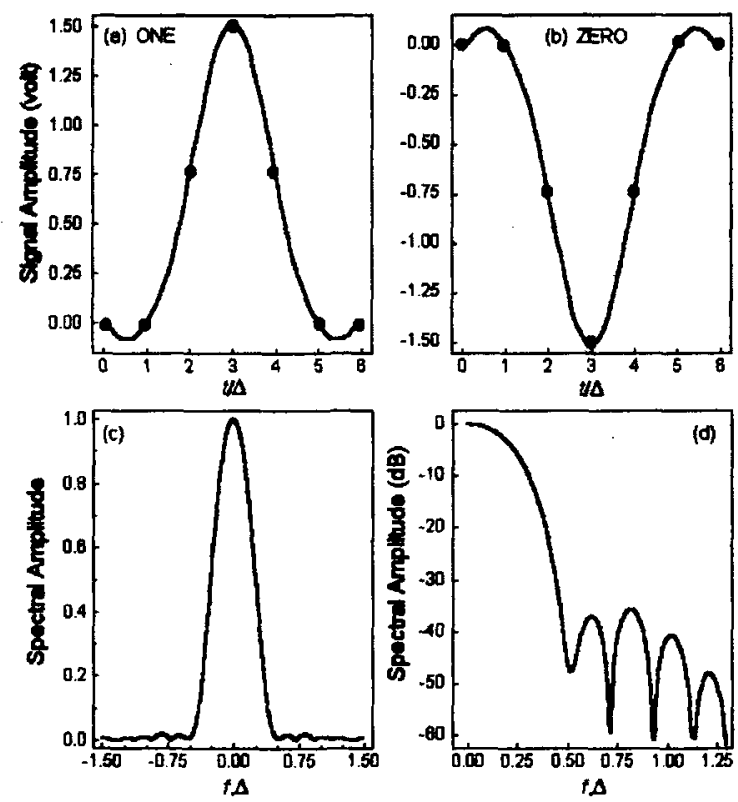

Figure 5. (a, b) Examples of waveforms that represent One and Zero in an ideal readout signal. The time-scale of the horizontal axis is normalized by the clock period $\Delta .(c, d)$ Fourier transform of the One/Zero waveform shown in (a, b). The frequency scale is normalized by $1 / \Delta$. The graph in (d) is the positive-frequency half of the spectrum shown in (c), plotted on a logarithmic scale in decibel units. design phase, the One/Zero functions should be adjusted until their (common) spectrum becomes band-limited and closely resembling the channel's spectrum of signal-tonoise ratio, $\operatorname{SNR}(f)$.

Once the One/Zero waveforms have been selected, the designer must choose a block-length $N$ for the sequence of channel-bits to be recorded as a single block on the storage medium. For instance, if $N=20$, one out of $2^{20}=1,048,576$ patterns will be written to the disk, each expected to yield an individual 20-bit-block waveform upon readout. The next step involves forming all $2^{N}$ possible waveforms that one expects to obtain upon reading an $N$-bit-block. This is done by shifting along the horizontal axis the One/Zero waveforms in multiples of the clock period $\Delta$, then adding the shifted functions in accordance with the particular $N$-bit sequence under consideration. For the One/Zero waveforms of Fig.5, which are $6 \Delta-$ long, the total length of the $N$-bit waveform will be $(N+5) \Delta$; a few such waveforms for the case of $N=20$ are shown in Fig.6.

\subsection{Genetic write-sequence-design algorithm}

The next task is to develop a writing strategy that would lead to recorded "patterns" on the disk which, upon readout, would yield these ideal waveforms. This can be done by a so-called "genetic algorithm." Each of the $2^{N}$ read waveforms corresponding to a "pattern" on the disk must be recorded using a sequence of laser pulses with several adjustable parameters. Typically, these parameters include the duration and height of each pulse, the width of the cooling pulse following each heating pulse, the interval and the laser power level between adjacent pulses, and so on. All these parameters should be listed in a table and considered adjustable at first. There will be $2^{N}$ rows in the table, each row containing all the parameters needed to record a single pattern on the medium that corresponds to a specific read waveform. The goal of the automatic search procedure outlined below is to find optimum values for each and every set of such parameters.

Step 1. Select one row from the table and assign a random set of values to the various write parameters. Record the random pulse sequence along a track. Next, read the
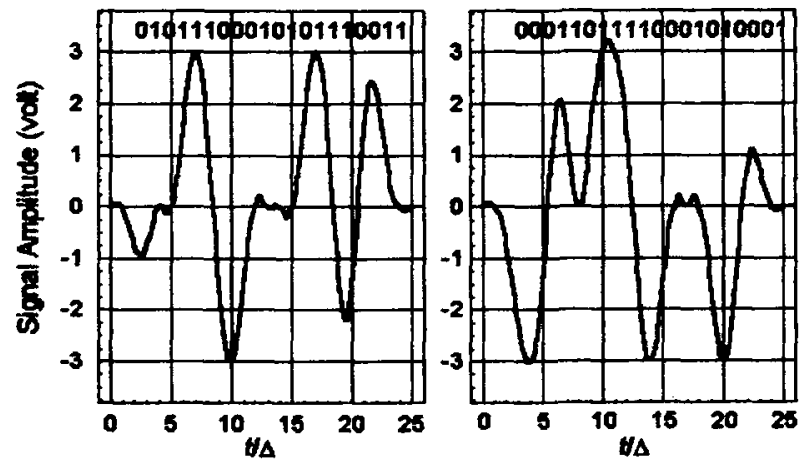

Figme 6. Typical read waveforms representing 20-bit blocks constructed from the One/Zero waveforms of Fig.5. All waveforms are $25 \Delta$-long, range from +3 to -3 volts, and start and stop at the mid-point of their range ( 0 volt). When these functions are sampled at integer multiples of $\Delta$ they yield the sample values $S(n \Delta)=0, \pm 0.75, \pm 1.5, \pm 2.25$, and \pm 3 volts. 
recorded pattern and, when applicable, average the readout signals over multiple recordings to reduce noise. Compare the ideal (desired) waveform for the selected row with the actual (averaged) signal thus obtained.

Step 2. Introduce small, random variations (mutations) in each of the parameters of the write sequence. Record the sequence again (as in Step 1) and compare the resulting read signal with the desired waveform. If the agreement between the two waveforms improves as a result of a particular mutation, accept that mutation, otherwise reject it (survival of the fittest).

Step 3. Repeat Step 2 until a stable set of parameters is obtained, that is, until further adjustments to the write sequence become ineffectual in improving the read waveform, and all subsequent mutations are rejected. At this point the write sequence should be yielding a readout signal that is nearly identical to the desired waveform.

Step 4. Repeat the above steps for another row in the table until all rows are exhausted.

Clearly the above algorithm can be much improved if the initial values of the parameters and the mutations are not chosen blindly, but in accordance with some expert knowledge of the recording process. Nonetheless, even a purely random strategy is expected to converge, under selection pressures, to a good writing scheme for each and every $N$-bit sequence. Thus, developing the write strategy might require several hours on an automated system, but this procedure needs to be performed only once during the development cycle of a given product. When all the adjustable parameters have been fixed at their optimal values, the complete lookup table should be programmed into a ROM chip and placed in the disk drives.

\subsection{Experiments}

Based on the above considerations we tried to design write sequences for each read waveform. To save time we avoided designing the entire write sequence by the genetic algorithm. Our preliminary algorithm is as follows:

Step 1. The target read waveform is divided into peak and valley segments. A set of unique write pulses is assigned in accordance with height/width of each peak and valley segment. After the first writing, the read waveform is compared with the target waveform.

Step 2. If the difference exceeds a certain threshold, each write pulse is randomly shifted within $\pm \Delta$. Record, read and compare the waveforms again. If the difference is still above the threshold, the laser power level for each pulse is randomly shifted by $\pm 5 \%$.

Step 3. Repeat steps 2 until stabilized.

This optimization algorithm was implemented in software that controls the write/read operations of our dynamic testbed. Experimental setup conditions were the same as described in the preceding section, but the disk was a commercial DVD-R/W disk. (This was suitable for automated experiments because pre-pit header areas are absent.) Fig.7 shows typical target and experimental readout waveforms. On the left-hand side the write pulses were optimized automatically by the above algorithm, whereas on the right-hand side they were manually optimized on the basis of expert knowledge. There are
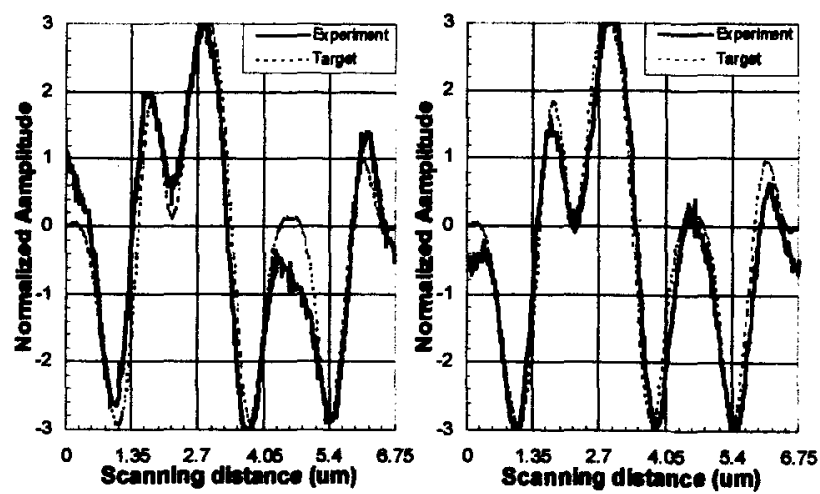

Figure 7. Theoretical target and the corresponding experimentally obtained read waveform. (Left) Write pulses were automatically optimized by the algorithm described in the text. (Right) Write pulses were manually optimized on the basis of expert knowledge.

still differences between target and the experimental waveform, and the automatic optimization routine needs refining, but we may conclude that the procedure is suitable for designing the write sequences. Although detection experiments have not yet been performed, experience with PRML signaling gives us confidence that there will be no major hurdles when we extract the encoded data from the retrieved patterns.

\section{Concluasions}

We proposed new optical recording methods based on the concept of reading "analog waveforms" from recorded amorphous/crystalline patterns. In the experiments using a phase-change disk, we obtained a variety of readout waveforms that were visually distinguishable. Although achieved improvement of the recording density was a factor of 1.5 using a DVD-RAM/2.6GB disk, we have not yet sufficiently confirmed the goal of superior density. However, we believe that abandoning the constraint of recording "perfect marks" on the media is a significant advantage for optical recording and leads to higher density. Farther improvements in the write process stability, optimized media, and detection algorithms should yield superior analog schemes.

Acknowledgements; The authors are grateful to $\mathrm{Dr}$. Chubing Peng of the Univ. of Arizona for experimental setup. This work has been supported by the U.S. Department of Commerce through the NSIC/MORE program (cooperative agreement no. 70NANB7H3054).

\section{References}

1) See, for example, G. Bouwhuis et al, Principles of Optical Disc Systems, Chapter 7, Adam Hilger, Bristol (1985).

2) S. Kobayashi et al, Tech. Dig. of Optical Data Storage Conference, p. 130 (1994).

3) M.Arai et al, Tech. Dig. of Joint MORIS/ISOM, p.32 (1997).

4) T. Ohta et al, Jpn. J. Appl. Phys., Vol. 39, p.770 (2000).

5) M.P. ONeill et al, Tech. Dig. of Optical Data Storage Conference, p. $170(2000)$.

6) M. Mansuripur et al, Apl..Opt., 36, No.35, p.9296(1997). 